\title{
NOUVELLE
}

\section{Synthèse totale de I'hydrocortisone dans la levure de boulanger}

\author{
Bruno Dumas, Roberto Spagnoli
}

B. Dumas: Functional

Genomics, Aventis,

13, quai Jules Guesdes,

94400 Vitry-sur-Seine, France.

R. Spagnoli : LDT

(Lead Discovery Technologies),

Aventis, 102, route de Noisy,

93235 Romainville, France.

Bruno.Dumas@aventis.com
> La levure s'ennuyait: on ne lui demandait, depuis des millénaires, que des tâches de routine, du pain, du vin, de la bière... Dans des temps plus récents, c'est vrai, l'homme, son ami, l'avait mise au défi de fabriquer pour lui des substances plus nobles (encore que, question noblesse, cela était loin de faire l'unanimité), des protéines que l'homme lui-même et les animaux ne savaient fabriquer qu'en petite quantité... Qui lui demanda des cytokines, qui des antigènes, qui de l'hirudine... Mais cela n'était pas à la hauteur de ses ambitions; au fond, un procaryote tout bête, un Escherichia coli minable, avait pu, souvent, réussir les mêmes choses. La levure rêvait d'être un jour appelée à une grande entreprise.

La nuit, elle lisait et relisait un texte ${ }^{l}$ où une phrase l'avait particulièrement frappée et chatouillait agréablement son ego: «Microorganisms can and will do everything, they are smarter, wiser, more energetic than chemists, engineers and others».

Quand, un beau jour ensoleillé de l'automne 1991, des biotechnologistes d'une entreprise romainvilloise, accompagnés d'autres éminents collègues de Strasbourg et de Gif-sur-yvette ${ }^{2}$ lui demandèrent de l'aide pour fabriquer des tonnes d'hydrocortisone à un prix défiant toute concurrence, $S$. cerevisiae n'hésita pas un instant: «Je suis prête - dit-elle simplement - les P450, je m'y

${ }^{1}$ D. Perlman: The laws of applied microbiology, 1979.

${ }^{2}$ Roussel Uclaf en coopération avec Transgène et le Centre de génétique moléculaire du Cnrs. connais». Ainsi commença l'aventure du projet $\mathrm{UCl}^{3}$.

L'hydrocortisone est encore, près de cinquante ans après sa mise sur le marché, un produit largement utilisé, soit comme médicament, soit comme intermédiaire pour la synthèse d'un principe actif. Son activité biologique est principalement liée à ses propriétés anti-inflammatoires. Ses effets secondaires sont connus et maîtrisés. La production mondiale approche la centaine de tonnes par an, une preuve de son succès lorsque l'on sait que la dose active chez l'homme est de l'ordre de quelques milligrammes parkg de poids.

La synthèse d'hydrocortisone actuellement exploitée chez Aventis (originellement mise en œuvre à Roussel-Uclaf) comprend huit étapes: sept sont chimiques et une est une bioconversion. II s'agit d'une nouvelle approche, très performante, qui a depuis quelques années remplacé le procédé précédent, beaucoup plus long (vingt étapes). Mais, dans un marché mondial à la concurrence acharnée, il nous fallait imaginer des procédés encore plus performants: c'est pourquoi nous nous sommes proposés, en nous inspirant de la merveilleuse efficacité de la biosynthèse d'hydrocortisone qui a lieu quotidiennement dans les surrénales de tout mammifère, de transférer la totalité de cette voie de synthèse dans un micro-organisme, en utilisant des techniques de biologie moléculaire. La voie naturelle comprend cinq étapes

${ }^{3}$ Universal corticosteroid intermediate. enzymatiques, dont quatre sont assurées par des enzymes à cytochromes P450, qui transforment le cholestérol, substrat très bon marché, en cette hormone glucocorticoïde très précieuse (en vert sur le schéma de biosynthèse) (Figure 1).

Peu de micro-organismes semblaient en 1991 à même de permettre la réalisation d'un tel projet. Finalement, notre choix s'est arrêté sur la levure de boulanger, S. cerevisiae, qui, sur le plan industriel et technologique, présentait à nos yeux de nombreux atouts, notamment celui d'être facilement fermentable à grande échelle, tout en étant sans danger (il est en fait classé GRAS, generally regarded as safe) et dont la manipulation génétique est aisée grâce à des outils de génie génétique et de génétique classique bien développés (entre-temps, en 1997 le génome de la levure a été totalement décrypté). Pour finir, la levure, micro-organisme eucaryote, possède des compartiments subcellulaires semblables à ceux qui existent naturellement dans les cellules des mammifères. Pour réussir à obtenir une levure qui exprime ces cinq étapes d'une façon fonctionnelle et coordonnée, nous avons dû surmonter de nombreuses difficultés: un minimum de huit protéines hétérologues doivent être exprimées dans une seule cellule; il faut aussi qu'un substrat adéquat soit fourni de façon endogène ou exogène (Figure 1, ersatz de cholestérol) à la première enzyme intervenant dans la voie de biosynthèse, la CYPllAl (ou P450scc), impliquée dans le clivage 


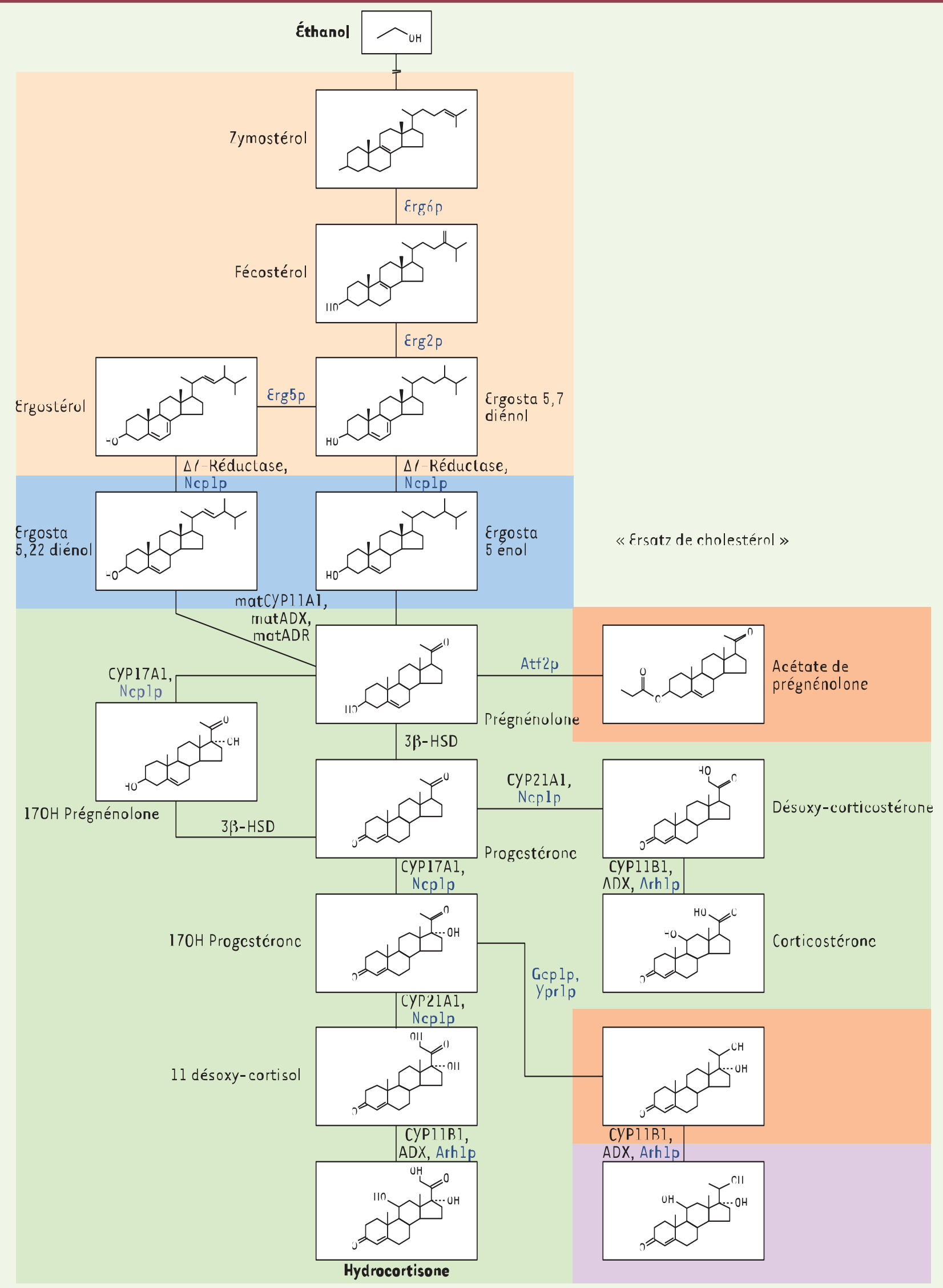


de la chaîne latérale du cholestérol, ce qui le transforme en prégnénolone; les P450 mitochondriaux et les transporteurs d'électrons associés doivent être ciblés dans les bons compartiments; il est essentiel que le flux métabolique de la voie de biosynthèse artificielle soit bien équilibré; enfin, il nous a fallu identifier et éliminer des réactions parasites, tout en prenant en compte la toxicité de certains intermédiaires pour la levure (voir Figure 1) [1]. Deux premières expériences décisives ont montré d'une part que la levure pouvait produire des substrats de la CYPllAl (Figure 1, ersatz de cholestérol) [2], d'autre part qu'elle possédait dans ses mitochondries un transporteur d'électrons (Figurel, Arhlp) compatible avec la CyPliBl ( $P 45011$ b responsable de l'hydroxylation en 11 du 11-désoxycortisol) [3]. De plus, d'autres expériences ont prouvé qu'il était possible de réaliser la partie centrale des étapes de la voie naturelle (de la prégnénolone à la $170 \mathrm{H}$-progestérone, Figure 1) en utilisant des substrats exogènes [4]. Les nouvelles avancées se sont succédées avec l'identification des gènes responsables de réactions parasites, notamment l'acétylation en 3 de la prégnénolone [5], puis de la réduction de la cétone en 20 de la 17-hydroxyprogestérone (voir Figure 1) [6].

II ne restait plus, si l'on peut dire, qu'à rendre la levure 13 fois transgénique... Le résultat final est à la hauteur des espérances, c'est-à-dire des souches de levure qui surexpriment 9 protéines dont 8 hétérologues après 13 modifications de leur génome, entraînant une production d'hydrocortisone substantielle (de l'ordre de $10 \mathrm{mg} /$ l dans le meilleur des cas) et totalement autonome [6]. Nous avons donc couplé la synthèse endogène de l'ergostérol de levure (Figure 1, schématisée en jaune) avec une voie de biosynthèse hétérologue (schématisée en vert sur la Figure 1) en utilisant une enzyme de plante, la $D 7$ réductase de A.thaliana comme charnière entre les deux voies de biosynthèse. Les voies parasites résultant de l'action des gènes de levure GCY1, YPRI et ATF2 ont été éliminées (en orange et en violet sur la Figure 1). Le flux est bien équilibré vers la production d'hydrocortisone, qui représente jusqu'à $70 \%$ des stéroïdes produits [6]. Le nouveau défi auquel nous sommes à présent confrontés est celui de l'augmentation de la qualité et de la quantité d'hydrocortisone produite pour satisfaire des impératifs de rentabilité industrielle.

Ce projet a donné lieu à des brevets, publications, communications multiples; le progrès scientifique qu'il représente aujourd'hui et, comme nous l'espérons, son succès industriel de demain, reposent largement sur l'enthousiasme et la créativité d'une équipe pluridisciplinaire, assistée d'un microorganisme intelligent et serviable. $\diamond$ Biosynthesis of hydrocortisone in yeast

\section{RéFÉRENCES}

1. Duport C, Schoepp B, Chatelain $\varepsilon$, Spagnoli R, Dumas B, Pompon D. Critical role of the plasma membrane for expression of mammalian mitochondrial side chain cleavage activity in yeast. EurJ Biochem 2003; 270: 1502-14.

2. Duport C, Spagnoli R, Degryse $\varepsilon$, Pompon D. Selfsufficient biosynthesis of pregnenolone and progesterone in engineered yeast. Nat Biotechnol 1998; 16: 186-9.

3. Lacour T, Achstetter T, Dumas B. Characterization of recombinant adrenodoxin reductase homologue (Arhlp) from yeast. Implication in in vitro cytochrome p4501lbeta monooxygenase system. J Biol Chem 1998; 273: 23984-92.
4. Degryse $\varepsilon$, Cauet $G$, Spagnoli R, Achstetter T. Pregnenolone metabolized to 17alphahydroxyprogesterone in yeast: biochemical analysis of a metabolic pathway. J Steroid Biochem Mol Biol 1999; 71: 239-46.

5. Cauet $G$, Degryse $\varepsilon$, Ledoux C, Spagnoli R, Achstetter T. Pregnenolone esterification in Saccharomyces cerevisiae. A potential detoxification mechanism. Eur J Biochem 1999; 261: 317-24.

6. Szczebara FM, Chandelier C, Villeret C, et al. Total biosynthesis of hydrocortisone from a simple carbon source in yeast. Nat Biotechnol 2003; 21 : 143-9.

Figure 1. Voie de biosynthèse de la levure recombinante. La voie de biosynthèse de l'ergostérol est schématisée en jaune, la voie de biosynthèse de l'hydrocortisone en vert, la charnière constituée par la D7 réductase en bleu. Les voies secondaires parasites sont représentées par les autres couleurs. ADX: adrénodoxine; ADR: adrénodoxine réductase; Arhlp: ADR related homolog; Atf2p: alcohol 0 acetyl transferase; CyPliAl: P450scc, side chain cleaving; CYP11B1: P45011b hydroxylase; CYP17Al: P45017a hydroxylase; CYP21A1: P450c21 hydroxylase; Erg2 : stérol C8-C7 isomérase; Erg5p: D22 désaturase; Erg6p: S-adénosylméthionine delta-24-stérol-C-méthyltransférase; Gcylp,Yprlp: aldoketo-reductase; 3bHSD : 3b hydroxy stéroïde déshydrogénase; Ncplp: NADPH P450 réductase. 